\title{
Breast Irradiation
}

National Cancer Institute

\section{Source}

National Cancer Institute. Breast Irradiation. NCI Thesaurus. Code C116438.

Localized radiation therapy to the breast. 